Modern Physics Letters A

(C) World Scientific Publishing Company

\title{
A Note on Supersymmetries in $\mathrm{AdS}_{5} / \mathrm{CFT}_{4}$
}

\author{
Fumiya Takeuchi \\ Graduate School of Science and Engineering, Ibaraki University, Mito 310-8512, Japan \\ 15nd404g@vc.ibaraki.ac.jp \\ Makoto Sakaguchi \\ Department of Physics, Ibaraki University, Mito 310-8512, Japan \\ makoto.sakaguchi.phys@vc.ibaraki.ac.jp
}

Received (Day Month Year)

Revised (Day Month Year)

\begin{abstract}
The $\mathcal{N}=4$ superconformal algebra is derived from the symmetry transformations of fields in the $\mathcal{N}=4 \mathrm{SYM}$ action in $D=4$. We use a Majorana-Weyl spinor in $D=10$ instead of four Weyl spinors in $D=4$. This makes it transparent to relate generators of the $\mathcal{N}=4$ superconformal algebra to those of the super- $\mathrm{AdS}_{5} \times \mathrm{S}^{5}$ algebra. Especially, we obtain the concrete map from the supersymmetries $Q$ and conformal supersymmetries $S$ in $\mathcal{N}=4 \mathrm{SYM}$ to the supersymmetries $\left(\mathcal{Q}_{1}, \mathcal{Q}_{2}\right)$ in the $\operatorname{AdS}_{5} \times \mathrm{S}^{5}$ background.
\end{abstract}

Keywords: super-Yang-Mills; superconformal symmetry; AdS/CFT.

PACS Nos.: 11.25.Tq; 11.30.-j; 12.60.Jv

\section{Introduction}

The gauge/gravity duality ${ }^{1}$ continues to attract much interest as it may provide hints for the non-perturbative formulation of M-theory and superstring theories. The AdS/CFT duality ${ }^{2,3}$ has been investigated as a concrete realization of it. A typical example is the $\mathrm{AdS}_{5} / \mathrm{CFT}_{4}$ duality which is a duality between the IIB superstring theory in the $\mathrm{AdS}_{5} \times \mathrm{S}^{5}$ background and the $\mathcal{N}=4$ super Yang-Mills (SYM) theory in $D=4$. It is noted that both the super-isometry algebra of the $\operatorname{AdS}_{5} \times \mathrm{S}^{5}$ background and the superconformal algebra of the $\mathcal{N}=4 \mathrm{SYM}$ are $\operatorname{psu}(2,2 \mid 4)$. This gives a fundamental non-trivial check for this duality.

It is known that the $\mathcal{N}=4 \mathrm{SYM}$ action in $D=4$ may be obtained as a dimensional reduction from the $\mathcal{N}=(1,0)$ SYM action in $D=10$. It follows that four Weyl spinors in $D=4$ can be represented by a $D=10$ Majorana-Weyl (MW) spinor. We use the $D=10 \mathrm{MW}$ spinor throughout this paper. One of the advantages of using the $D=10 \mathrm{MW}$ spinor notation is to make it transparent to explore supersymmetries in $\mathrm{AdS}_{5} / \mathrm{CFT}_{4}$.

In this note, we will derive the concrete transformation laws of fields in the 
$\mathcal{N}=4 \mathrm{SYM}$ under the $\operatorname{PSU}(2,2 \mid 4)$ in the $D=10 \mathrm{MW}$ spinor notation. It is shown that the supersymmetry charge $Q$ is a MW spinor of positive chirality while the conformal supersymmetry charge $S$ is that of negative chirality. In the $\mathrm{AdS}_{5} / \mathrm{CFT}_{4}$ duality, these must be related to the supersymmetry charges $\left(\mathcal{Q}_{1}, \mathcal{Q}_{2}\right)$ of positive chirality on the $\operatorname{AdS}_{5} \times S^{5}$ background. The thrust of this note is to give a concrete map from the generators of the $\mathcal{N}=4$ superconformal algebra to those of the super- $\mathrm{AdS}_{5} \times \mathrm{S}^{5}$ algebra, including fermionic charges.

In the next section, we will begin with deriving transformation laws of the $\mathcal{N}=$ $(1,0)$ super-Poincaré symmetry by examining the SYM action in $D=10$. It is shown in section 3 that they reduce to the $D=4$ Poincaré symmetry, SU(4) Rsymmetry and 16 supersymmetries $Q$ of the SYM action in $D=4$. Furthermore the $\mathrm{SYM}$ in $D=4$ is shown to acquire extra enhanced symmetries: special conformal symmetry, scale symmetry and 16 conformal supersymmetries $S$, so that it has the $D=4, \mathcal{N}=4$ superconformal symmetry in total. In section 4 , we will give a concrete map between the superconformal algebra in $D=4$ and the supersymmetry algebra of $\mathrm{AdS}_{5} \times \mathrm{S}^{5} .{ }^{4}$ Our map shows that the conformal supersymmetries $S$ are the supersymmetries which are broken in the presence of a D3-brane and are restored in the near horizon limit, as expected. In the final section, we comment on the central charges realized in terms of the $\mathcal{N}=4$ SYM fields.

\section{2. $\mathcal{N}=(1,0)$ super Yang-Mills theory in $D=10$}

We will show that the action of the $D=10 \mathrm{SYM}$ has the $\mathcal{N}=(1,0)$ super-Poincaré symmetry in $D=10$. The $\mathcal{N}=(1,0) \mathrm{SYM}$ action we examine is given as

$$
S=\int \mathrm{d}^{10} x\left[-\frac{1}{4} F_{M N}^{a} F^{a M N}-\frac{i}{2} \bar{\psi}^{a} \Gamma^{M} D_{M} \psi^{a}\right]
$$

where we have defined the following objects

$$
F_{M N}^{a}=\partial_{M} A_{N}^{a}-\partial_{N} A_{M}^{a}+g f^{a b c} A_{M}^{b} A_{N}^{c}, \quad D_{M} \psi^{a}=\partial_{M} \psi^{a}+g f^{a b c} A_{M}^{b} \psi^{c}
$$

and $M, N=0,1, \cdots, 9$ denote spacetime indices. The fields $A_{M}=A_{M}^{a} T^{a}$ and $\psi=\psi^{a} T^{a}$ transform in the adjoint representation of the gauge group $\mathrm{U}(N)$ where $N \times N$ matrices $T^{a}\left(a=1,2, \cdots, N^{2}\right)$ are the generators of $\mathrm{U}(N)$ satisfying $\left[T^{a}, T^{b}\right]=i f^{a b c} T^{c}$. The Gamma-matrices $\Gamma^{M}$ in $(1+9)$-dimensions are $32 \times 32$ matrices satisfying $\left\{\Gamma^{M}, \Gamma^{N}\right\}=2 \eta^{M N}$ where $\eta^{M N}=\operatorname{diag}(-1,+1, \cdots,+1)$. The $\psi^{a}$ are MW spinors in $(1+9)$-dimensions which are 32 component column vectors satisfying $h_{+} \psi^{a}=\psi^{a}$, where we have defined $h_{ \pm} \equiv \frac{1}{2}\left(1 \pm \Gamma_{11}\right)$ with $\Gamma_{11}=\Gamma^{0123 \cdots 9}$. We define $\bar{\psi}^{a} \equiv \psi^{a T} C$ where $C$ is the charge conjugation matrix satisfying $C \Gamma_{M} C^{-1}=-\Gamma_{M}^{T}$, $C^{\dagger} C=1$ and $C^{T}=-C$.

The action (1) is invariant under the following symmetries. ${ }^{\text {a }}$

aThe superscript on $\delta$ denotes the parameter of the corresponding transformation. 
- Poincaré symmetry generated by the translation $P_{M}$ with a parameter $a^{M}$

$$
\delta_{P}^{a} A_{M}^{a}=a^{N} \partial_{N} A_{M}^{a}, \quad \delta_{P}^{a} \psi^{a}=a^{N} \partial_{N} \psi^{a},
$$

and the Lorentz rotation $L_{M N}$ with a parameter $\omega^{M N}=-\omega^{N M}$

$$
\delta_{L}^{\omega} A_{M}^{a}=x^{P} \omega_{P}^{N} \partial_{N} A_{M}^{a}+\omega_{M}^{N} A_{N}^{a}, \quad \delta_{L}^{\omega} \psi^{a}=x^{P} \omega_{P}^{N} \partial_{N} \psi^{a}+\frac{1}{4} \omega^{M N} \Gamma_{M N} \psi^{a} .
$$

- $\mathcal{N}=(1,0)$ supersymmetry generated by the supertranslation $Q$ with a Grassmann-odd MW spinor parameter $\epsilon$ of positive chirality $h_{+} \epsilon=\epsilon$

$$
\delta_{Q}^{\epsilon} A_{M}^{a}=\frac{i}{2} \bar{\epsilon} \Gamma_{M} \psi^{a}, \quad \delta_{Q}^{\epsilon} \psi^{a}=-\frac{1}{4} F_{M N}^{a} \Gamma^{M N} \epsilon .
$$

A key relation for this symmetry is the Fierz identity

$$
\left(C \Gamma_{M}\right)_{\alpha(\beta}\left(C \Gamma^{M}\right)_{\gamma \delta)}=0,
$$

which is proven in Appendix A as (A.3).

- Gauge symmetry generated by a local gauge transformation with a parameter $\lambda^{a}(x)$

$$
\delta_{g}^{\lambda} A_{M}^{a}=D_{M} \lambda^{a}(x)=\partial_{M} \lambda^{a}+g f^{a b c} A_{M}^{b} \lambda^{c}, \quad \delta_{g}^{\lambda} \psi^{a}=g f^{a b c} \psi^{b} \lambda^{c} .
$$

\section{1. $\mathcal{N}=(1,0)$ super-Poincaré symmetry in $D=10$}

We shall derive the symmetry algebra of the above symmetries. For this purpose, we need to know commutators among $\left\{\delta_{L}, \delta_{P}, \delta_{Q}\right\}$. Commutators among $\left\{\delta_{L}, \delta_{P}\right\}$ are obtained as

$$
\begin{aligned}
{\left[\delta_{P}^{a}, \delta_{P}^{a^{\prime}}\right] \Phi } & =0, \\
{\left[\delta_{L}^{\omega}, \delta_{P}^{a}\right] \Phi } & =\delta_{P}^{\tilde{a}} \Phi \quad \text { with } \quad \tilde{a}^{M}=a^{N} \omega_{N}{ }^{M} \\
{\left[\delta_{L}^{\omega}, \delta_{L}^{\omega^{\prime}}\right] \Phi } & =\delta_{L}^{\tilde{\omega}} \Phi \quad \text { with } \quad \tilde{\omega}_{M}{ }^{N}=\omega_{M}^{\prime}{ }^{P} \omega_{P}{ }^{N}-\omega_{M}{ }^{P} \omega_{P}^{\prime}{ }^{N} .
\end{aligned}
$$

In this section, we will denote collectively the fields $A_{M}^{a}$ and $\psi^{a}$ simply as $\Phi$. Next, we examine commutators between $\delta_{Q}$ and one of $\left\{\delta_{L}, \delta_{P}, \delta_{Q}\right\}$. One finds

$$
\begin{aligned}
& {\left[\delta_{Q}^{\epsilon}, \delta_{Q}^{\epsilon^{\prime}}\right] \Phi=\delta_{P}^{\tilde{a}} \Phi+\delta_{g}^{\lambda} \Phi \quad \text { with } \tilde{a}^{N}=-\frac{i}{2} \bar{\epsilon} \Gamma^{N} \epsilon^{\prime} \text { and } \lambda^{a}=\frac{i}{2} \bar{\epsilon} \Gamma^{N} \epsilon^{\prime} A_{N}^{a},} \\
& {\left[\delta_{Q}^{\epsilon}, \delta_{P}^{a}\right] \Phi=0,} \\
& {\left[\delta_{Q}^{\epsilon}, \delta_{L}^{\omega}\right] \Phi=\delta_{Q}^{\tilde{\epsilon}} \Phi \quad \text { with } \quad \tilde{\epsilon}=\frac{1}{4} \omega^{M N} \Gamma_{M N} \epsilon .}
\end{aligned}
$$

In eqn (11), we have used the equation of motion

$$
\Gamma^{M} D_{M} \psi^{a}=0
$$

and the Fierz identity (6).

We may define generators $Q_{\alpha}, P_{M}$ and $L_{M N}$ by

$$
\delta_{Q}^{\epsilon} \Phi=-i\left[Q_{\alpha} \epsilon^{\alpha}, \Phi\right], \quad \delta_{P}^{a} \Phi=-i\left[a^{N} P_{N}, \Phi\right], \quad \delta_{L}^{\omega} \Phi=-i\left[\frac{1}{2} w^{M N} L_{M N}, \Phi\right],
$$


Fumiya Takeuchi and Makoto Sakaguchi

where $Q$ is a $D=10 \mathrm{MW}$ spinor of positive chirality $Q h_{+}=Q$, and a 32-component row vector. On the right hand side of $\left[\delta_{Q}^{\epsilon}, \delta_{Q}^{\eta}\right]$, there appears a gauge transformation $\delta_{g}$. This is because $A_{M}^{a}$ and $\psi^{a}$ are not gauge invariant. So we may omit $\delta_{g} \Phi$ in considering spacetime symmetry algebra. Let us consider the equation $\left[\delta_{Q}^{\epsilon}, \delta_{Q}^{\epsilon^{\prime}}\right] \Phi=$ $\delta_{P}^{\tilde{a}} \Phi$. The left hand side means

$$
\left[\delta_{Q}^{\epsilon}, \delta_{Q}^{\epsilon^{\prime}}\right] \Phi=\left[Q \epsilon,\left[Q \epsilon^{\prime}, \Phi\right]\right]-\left[Q \epsilon^{\prime},[Q \epsilon, \Phi]\right]=\left[\left[Q \epsilon, Q \epsilon^{\prime}\right], \Phi\right],
$$

while the right hand side means $\delta_{P}^{\tilde{a}} \Phi=-i\left[\tilde{a}^{N} P_{N}, \Phi\right]$. It follows from these expressions that

$$
\left[Q \epsilon, Q \epsilon^{\prime}\right]=-i \tilde{a}^{N} P_{N}
$$

Furthermore, since

$$
\begin{aligned}
& {\left[Q \epsilon, Q \epsilon^{\prime}\right]=-\epsilon^{\alpha}\left\{Q_{\alpha}, Q_{\beta}\right\} \epsilon^{\prime \beta},} \\
& -i \tilde{a}^{N} P_{N}=-i\left(-\frac{i}{2} \bar{\epsilon} \Gamma^{N} \epsilon^{\prime}\right) P_{N}=-\epsilon^{\alpha} \frac{1}{2}\left(C \Gamma^{N}\right)_{\alpha \beta} P_{N} \epsilon^{\prime \beta},
\end{aligned}
$$

we obtain

$$
\left\{Q_{\alpha}, Q_{\beta}\right\}=\frac{1}{2}\left(C \Gamma^{N} h_{+}\right)_{\alpha \beta} P_{N} .
$$

Similarly, $\left[\delta_{Q}^{\epsilon}, \delta_{L}^{\omega}\right] \Phi=\delta_{Q}^{\tilde{\epsilon}} \Phi,\left[\delta_{L}^{\omega}, \delta_{P}^{a}\right] \Phi=\delta_{P}^{\tilde{a}} \Phi$ and $\left[\delta_{L}^{\omega}, \delta_{L}^{\omega^{\prime}}\right] \Phi=\delta_{L}^{\tilde{\omega}} \Phi$ lead to

$$
\begin{aligned}
{\left[Q_{\alpha}, L_{M N}\right] } & =-\frac{i}{2}\left(Q \Gamma_{M N}\right)_{\alpha}, \quad\left[L_{M N}, P_{P}\right]=i\left(\eta_{N P} P_{M}-\eta_{M P} P_{N}\right), \\
{\left[L_{M N}, L_{P Q}\right] } & =i\left(\eta_{N P} L_{M Q}-\eta_{M P} L_{N Q}-\eta_{N Q} L_{M P}+\eta_{M Q} L_{N P}\right),
\end{aligned}
$$

respectively. Summarizing, we have shown that the action (1) is invariant under the $D=10, \mathcal{N}=(1,0)$ super-Poincaré symmetry (19) and (20).

\section{3. $\mathcal{N}=4$ super Yang-Mills theory in $D=4$}

\section{1. $D=4 S Y M$ from $D=10 S Y M$}

We consider a dimensional reduction to four-dimensions by assuming that

$$
M=(\mu, i), \quad A_{M}^{a}=\left(A_{\mu}^{a}\left(x^{\mu}\right), \phi_{i}^{a}\left(x^{\mu}\right)\right), \quad \psi^{a}=\psi^{a}\left(x^{\mu}\right)
$$

where $\mu=0,1,2,3$ and $i=4,5, \ldots, 9$. It is straightforward to see that the action (1) reduces to

$$
\begin{aligned}
S=\int \mathrm{d}^{4} x & {\left[-\frac{1}{4} F_{\mu \nu}^{a} F^{a \mu \nu}-\frac{1}{2} D_{\mu} \phi_{i}^{a} D^{\mu} \phi_{i}^{a}-\frac{1}{4} g^{2} f^{a b c} \phi_{i}^{b} \phi_{j}^{c} f^{a d e} \phi_{i}^{d} \phi_{j}^{e}\right.} \\
& \left.-\frac{i}{2} \bar{\psi}^{a} \Gamma^{\mu} D_{\mu} \psi^{a}-\frac{i}{2} \bar{\psi}^{a} \Gamma^{i} g f^{a b c} \phi_{i}^{b} \psi^{c}\right]
\end{aligned}
$$

where $F_{\mu \nu}^{a}, D_{\mu} \phi_{i}^{a}$ and $D_{\mu} \psi^{a}$ are defined by

$$
\begin{aligned}
F_{\mu \nu}^{a} & =\partial_{\mu} A_{\nu}^{a}-\partial_{\nu} A_{\mu}^{a}+g f^{a b c} A_{\mu}^{b} A_{\nu}^{c}, \quad D_{\mu} \phi_{i}^{a}=\partial_{\mu} \phi_{i}^{a}+g f^{a b c} A_{\mu}^{b} \phi_{i}^{c}, \\
D_{\mu} \psi^{a} & =\partial_{\mu} \psi^{a}+g f^{a b c} A_{\mu}^{b} \psi^{c} .
\end{aligned}
$$




\subsection{Symmetries inherited from $D=10 S Y M$}

The following symmetries are inherited from $D=10$ SYM. It is straightforward to see that the action (22) is invariant under these symmetry transformations. Here and hereafter we will denote collectively $A_{\mu}^{a}, \phi_{i}^{a}$, and $\psi^{a}$ simply as $\Phi$.

- Translation symmetry $P_{\mu}$ with a parameter $a^{\mu}$

$$
\delta_{P}^{a} \Phi=a^{\nu} \partial_{\nu} \Phi
$$

- Lorentz symmetry $L_{\mu \nu}$ with a parameter $\omega^{\mu \nu}=-\omega^{\nu \mu}$

$$
\begin{aligned}
\delta_{L}^{\omega} A_{\mu}^{a} & =x^{\sigma} \omega_{\sigma}^{\rho} \partial_{\rho} A_{\mu}^{a}+\omega_{\mu}^{\nu} A_{\nu}^{a}, \quad \delta_{L}^{\omega} \phi_{i}^{a}=x^{\sigma} \omega_{\sigma}^{\rho} \partial_{\rho} \phi_{i} \\
\delta_{L}^{\omega} \psi^{a} & =x^{\sigma} \omega_{\sigma}^{\rho} \partial_{\rho} \psi^{a}+\frac{1}{4} \omega^{\mu \nu} \Gamma_{\mu \nu} \psi^{a} .
\end{aligned}
$$

- $\mathrm{SO}(6)$ symmetry $R_{i j}$ with a parameter $m^{i j}=-m^{j i}$

$$
\delta_{R}^{m} A_{\mu}^{a}=0, \quad \delta_{R}^{m} \phi_{i}^{a}=m_{i}^{j} \phi_{j}^{a}, \quad \delta_{R}^{m} \psi^{a}=\frac{1}{4} m^{i j} \Gamma_{i j} \psi^{a} .
$$

- Supersymmetry $Q_{\alpha}$ with a Grassmann-odd MW spinor parameter $\epsilon$

$$
\begin{aligned}
\delta_{Q}^{\epsilon} A_{\mu}^{a} & =\frac{i}{2} \bar{\epsilon} \Gamma_{\mu} \psi^{a}, \quad \delta_{Q}^{\epsilon} \phi_{i}^{a}=\frac{i}{2} \bar{\epsilon} \Gamma_{i} \psi^{a}, \\
\delta_{Q}^{\epsilon} \psi^{a} & =-\frac{1}{4} F_{\mu \nu}^{a} \Gamma^{\mu \nu} \epsilon-\frac{1}{2} D_{\mu} \phi_{i}^{a} \Gamma^{\mu i} \epsilon-\frac{1}{4} g f^{a b c} \phi_{i}^{b} \phi_{j}^{c} \Gamma^{i j} \epsilon .
\end{aligned}
$$

The supersymmetry parameter $\epsilon$ has 16 nontrivial components, while a Weyl spinor in four-dimensions consists of two complex components. It follows that $\epsilon$ consists of four Weyl spinors in four-dimensions so that the action is $\mathcal{N}=4$ supersymmetric.

- Gauge symmetry with a parameter $\lambda^{a}(x)$

$$
\delta_{g}^{\lambda} A_{\mu}^{a}=D_{\mu} \lambda^{a}=\partial_{\mu} \lambda^{a}+g f^{a b c} A_{\mu}^{b} \lambda^{c}, \quad \delta_{g}^{\lambda} \phi_{i}^{a}=g f^{a b c} \phi_{i}^{b} \lambda^{c}, \quad \delta_{g}^{\lambda} \psi^{a}=g f^{a b c} \psi^{b} \lambda^{c} .
$$

The commutators among $\left\{\delta_{P}, \delta_{L}, \delta_{R}\right\}$ are found to be

$$
\begin{aligned}
{\left[\delta_{P}, \delta_{P}\right] \Phi } & =0, \\
{\left[\delta_{L}^{\omega}, \delta_{P}^{a}\right] \Phi } & =\delta_{P}^{\tilde{a}} \Phi \quad \text { with } \quad \tilde{a}^{\mu}=a^{\nu} \omega_{\nu}^{\mu} \\
{\left[\delta_{L}^{\omega}, \delta_{L}^{\omega^{\prime}}\right] \Phi } & =\delta_{L}^{\tilde{\omega}} \Phi \quad \text { with } \quad \tilde{\omega}_{\mu}{ }^{\nu}=\omega_{\mu}^{\prime} \omega_{\rho}{ }^{\nu}-\omega_{\mu}{ }^{\rho} \omega_{\rho}^{\prime \nu} \\
{\left[\delta_{R}^{m}, \delta_{R}^{m^{\prime}}\right] \Phi } & =\delta_{R}^{\tilde{m}} \Phi \quad \text { with } \quad \tilde{m}_{i}{ }^{j}=m_{i}^{\prime k} m_{k}{ }^{j}-m_{i}{ }^{k} m_{k}^{\prime}{ }^{j}
\end{aligned}
$$


Fumiya Takeuchi and Makoto Sakaguchi

while those including $\delta_{Q}$ are found as

$$
\begin{aligned}
& {\left[\delta_{Q}, \delta_{P}\right] \Phi=0} \\
& {\left[\delta_{Q}^{\epsilon}, \delta_{L}^{\omega}\right] \Phi=\delta_{Q}^{\tilde{\epsilon}} \Phi \quad \text { with } \quad \tilde{\epsilon}=\frac{1}{4} \omega^{\mu \nu} \Gamma_{\mu \nu} \epsilon} \\
& {\left[\delta_{Q}^{\epsilon}, \delta_{R}^{m}\right] \Phi=\delta_{Q}^{\tilde{\epsilon}} \Phi \quad \text { with } \quad \tilde{\epsilon}=\frac{1}{4} m^{i j} \Gamma_{i j} \epsilon} \\
& {\left[\delta_{Q}^{\epsilon}, \delta_{Q}^{\epsilon^{\prime}}\right] \Phi=\delta_{P}^{\tilde{a}} \Phi+\delta_{g}^{\lambda} \Phi \quad \text { with } \quad \tilde{a}^{\mu}=\frac{i}{2} \bar{\epsilon}^{\prime} \Gamma^{\mu} \epsilon \quad \text { and } \quad \lambda^{a}=-\frac{i}{2} A_{\mu}^{a} \bar{\epsilon}^{\prime} \Gamma^{\mu} \epsilon-\frac{i}{2} \phi_{i}^{a} \bar{\epsilon}^{\prime} \Gamma^{i} \epsilon}
\end{aligned}
$$

In order to evaluate $\left[\delta_{Q}^{\epsilon}, \delta_{Q}^{\epsilon^{\prime}}\right] \psi$ in (36), we have used the equation of motion

$$
\Gamma^{\mu} D_{\mu} \psi^{a}+g f^{a b c} \phi_{i}^{b} \Gamma^{i} \psi^{c}=0
$$

and the Fierz identity (6). As will be seen in section 3.4, they represent the $\mathcal{N}=4$ super-Poincaré symmetry.

\subsection{Enhanced symmetries}

In addition to the $\mathcal{N}=4$ super-Poincare symmetry inherited from the $D=10$ SYM, the $D=4$ SYM action (22) acquires the following enhanced symmetries: the special conformal symmetry $K_{\mu}$, the scale symmetry $D$ and the conformal supersymmetry $S_{\alpha}$.

Special conformal symmetry $K_{\mu}$

One can show that the $D=4 \mathrm{SYM}$ action (22) is invariant under the special conformal transformation $K_{\mu}$ with a parameter $b^{\mu}$

$$
\begin{aligned}
\delta_{K}^{b} A_{\mu}^{a} & =\delta x^{\nu} \partial_{\nu} A_{\mu}^{a}-2(b x) A_{\mu}^{a}+2\left(x_{\mu} b^{\nu}-b_{\mu} x^{\nu}\right) A_{\nu}^{a}, \\
\delta_{K}^{b} \phi_{i}^{a} & =\delta x^{\nu} \partial_{\nu} \phi_{i}^{a}-2(b x) \phi_{i}^{a}, \\
\delta_{K}^{b} \psi^{a} & =\delta x^{\nu} \partial_{\nu} \psi^{a}-3(b x) \psi^{a}+\frac{1}{2}\left(x^{\mu} b^{\nu}-x^{\nu} b^{\mu}\right) \Gamma_{\mu \nu} \psi^{a},
\end{aligned}
$$

where $\delta x^{\nu}=-2(b x) x^{\nu}+b^{\nu} x^{2}$ and $(b x)=b^{\mu} x_{\mu}$. Note that the second terms in the right hand sides denote $-2 \Delta(\Phi)(b x) \Phi$ where $\Delta(\Phi)$ stands for the dimension of $\Phi$, i.e. $\Delta\left(A_{\mu}^{a}\right)=\Delta\left(\phi_{i}^{a}\right)=1$ and $\Delta\left(\psi^{a}\right)=3 / 2$.

Commutators between $\delta_{K}^{b}$ and one of $\left\{\delta_{L}, \delta_{R}, \delta_{K}\right\}$ do not generate new transformations

$$
\begin{aligned}
{\left[\delta_{K}^{b}, \delta_{L}^{\omega}\right] \Phi } & =\delta_{K}^{\tilde{b}} \Phi \quad \text { with } \quad \tilde{b}^{\mu}=-b^{\nu} \omega_{\nu}^{\mu} \\
{\left[\delta_{K}, \delta_{K}\right] \Phi } & =0 \\
{\left[\delta_{K}, \delta_{R}\right] \Phi } & =0
\end{aligned}
$$

but $\left[\delta_{K}, \delta_{P}\right] \Phi$ and $\left[\delta_{K}, \delta_{Q}\right] \Phi$ are shown to require extra transformations $\delta_{D}$ and $\delta_{S}$. 


\section{Scale symmetry D}

The commutator between $\delta_{K}$ and $\delta_{P}$ is

$$
\left[\delta_{K}^{b}, \delta_{P}^{a}\right] \Phi=\delta_{L}^{\tilde{\omega}} \Phi-2 a^{\mu} b_{\mu}\left(x^{\nu} \partial_{\nu} \Phi+\Delta(\Phi) \Phi\right) \quad \text { with } \quad \tilde{\omega}^{\mu \nu}=2\left(a^{\mu} b^{\nu}-b^{\mu} a^{\nu}\right)
$$

From (44) we define the scale transformation $D$ with a parameter $\alpha$

$$
\delta_{D}^{\alpha} \Phi=\alpha\left(x^{\nu} \partial_{\nu} \Phi+\Delta(\Phi) \Phi\right)
$$

so that (44) turns to $\left[\delta_{K}^{b}, \delta_{P}^{a}\right] \Phi=\delta_{L}^{\tilde{\omega}} \Phi+\delta_{D}^{\tilde{\alpha}} \Phi$ with $\tilde{\alpha}=-2 a^{\mu} b_{\mu}$.

\section{Conformal supersymmetry $S_{\alpha}$}

We find that $\left[\delta_{K}^{b}, \delta_{Q}^{\epsilon}\right] \Phi$ takes the form

$$
\begin{aligned}
{\left[\delta_{K}^{b}, \delta_{Q}^{\epsilon}\right] A_{\mu}^{a}=} & -\frac{i}{2} \bar{\epsilon}(b \Gamma)(x \Gamma) \Gamma_{\mu} \psi^{a}, \\
{\left[\delta_{K}^{b}, \delta_{Q}^{\epsilon}\right] \phi_{i}^{a}=} & -\frac{i}{2} \bar{\epsilon}(b \Gamma)(x \Gamma) \Gamma_{i} \psi^{a}, \\
{\left[\delta_{K}^{b}, \delta_{Q}^{\epsilon}\right] \psi^{a}=} & \frac{1}{4} F_{\mu \nu} \Gamma^{\mu \nu}(x \Gamma)(b \Gamma) \epsilon+\frac{1}{2} D_{\mu} \phi_{i}^{a} \Gamma^{\mu i}(x \Gamma)(b \Gamma) \epsilon \\
& +\frac{1}{4} g f^{a b c} \phi_{i}^{b} \phi_{j}^{c} \Gamma^{i j}(x \Gamma)(b \Gamma) \epsilon-\phi_{i}^{a} \Gamma^{i}(b \Gamma) \epsilon
\end{aligned}
$$

From these expressions, we define the conformal supersymmetry transformation $S_{\alpha}$ with a parameter $\eta$

$$
\begin{aligned}
\delta_{S}^{\eta} A_{\mu}^{a} & =\frac{i}{2} \bar{\eta}(x \Gamma) \Gamma_{\mu} \psi^{a} \\
\delta_{S}^{\eta} \phi_{i}^{a} & =\frac{i}{2} \bar{\eta}(x \Gamma) \Gamma_{i} \psi^{a} \\
\delta_{S}^{\eta} \psi^{a} & =\frac{1}{4} F_{\mu \nu}^{a} \Gamma^{\mu \nu}(x \Gamma) \eta+\frac{1}{2} D_{\mu} \phi_{i}^{a} \Gamma^{\mu i}(x \Gamma) \eta+\frac{1}{4} g f^{a b c} \phi_{i}^{b} \phi_{j}^{c} \Gamma^{i j}(x \Gamma) \eta-\phi_{i}^{a} \Gamma^{i} \eta
\end{aligned}
$$

so that $\left[\delta_{K}^{b}, \delta_{Q}^{\epsilon}\right] \Phi=\delta_{S}^{\tilde{\eta}} \Phi$ with $\tilde{\eta}=(b \Gamma) \epsilon$. Note that $\eta$ has the opposite chirality to $\epsilon$ and $\psi$.

Nontrivial commutators including $\delta_{D}$ are

$$
\begin{aligned}
& {\left[\delta_{D}^{\alpha}, \delta_{P}^{a}\right] \Phi=\delta_{P}^{\tilde{a}} \Phi \quad \text { with } \quad \tilde{a}^{\mu}=\alpha a^{\mu},} \\
& {\left[\delta_{D}^{\alpha}, \delta_{K}^{b}\right] \Phi=\delta_{K}^{\tilde{b}} \Phi \quad \text { with } \quad \tilde{b}^{\mu}=-\alpha b^{\mu}} \\
& {\left[\delta_{D}^{\alpha}, \delta_{Q}^{\epsilon}\right] \Phi=\delta_{Q}^{\tilde{\epsilon}} \Phi \quad \text { with } \quad \tilde{\epsilon}=\frac{1}{2} \alpha \epsilon} \\
& {\left[\delta_{D}^{\alpha}, \delta_{S}^{\eta}\right] \Phi=\delta_{S}^{\tilde{\eta}} \Phi \quad \text { with } \quad \tilde{\eta}=-\frac{1}{2} \alpha \eta}
\end{aligned}
$$


Fumiya Takeuchi and Makoto Sakaguchi

Commutators including $\delta_{S}^{\eta}$ are found to be

$$
\begin{aligned}
& {\left[\delta_{S}^{\eta}, \delta_{L}^{\omega}\right] \Phi }=\delta_{S}^{\tilde{\eta}} \Phi \quad \text { with } \quad \tilde{\eta}=\frac{1}{4} \omega^{\mu \nu} \Gamma_{\mu \nu} \eta \\
& {\left[\delta_{S}^{\eta}, \delta_{R}^{m}\right] \Phi=\delta_{S}^{\tilde{\eta}} \Phi \quad \text { with } \quad \tilde{\eta}=\frac{1}{4} m^{i j} \Gamma_{i j} \eta } \\
& {\left[\delta_{P}^{a}, \delta_{S}^{\eta}\right] \Phi=\delta_{Q}^{\tilde{\epsilon}} \Phi \quad \text { with } \quad \tilde{\epsilon}=a^{\mu} \Gamma_{\mu} \eta } \\
& {\left[\delta_{S}^{\eta}, \delta_{S}^{\eta^{\prime}}\right] \Phi=\delta_{K}^{\tilde{b}} \Phi+\delta_{g}^{\lambda} \Phi } \\
& \text { with } \quad \tilde{b}^{\mu}=-\frac{i}{2} \bar{\eta} \Gamma^{\mu} \eta^{\prime} \quad \text { and } \quad \lambda^{a}=-\frac{i}{2} \bar{\eta}(x \Gamma)\left(\phi_{i}^{a} \Gamma^{i}+A_{\mu}^{a} \Gamma^{\mu}\right)(x \Gamma) \eta^{\prime}
\end{aligned}
$$

and

$$
\begin{aligned}
{\left[\delta_{Q}^{\epsilon}, \delta_{S}^{\eta}\right] \phi_{i}^{a} } & =\delta_{L}^{\omega} \phi_{i}^{a}+\delta_{D}^{\alpha} \phi_{i}^{a}+\delta_{R}^{m} \phi_{i}^{a}+\delta_{g}^{\lambda} \phi_{i}^{a}, \\
{\left[\delta_{Q}^{\epsilon}, \delta_{S}^{\eta}\right] A_{\mu}^{a} } & =\delta_{L}^{\omega} A_{\mu}^{a}+\delta_{D}^{\alpha} A_{\mu}^{a}+\delta_{g}^{\lambda} A_{\mu}^{a} \\
{\left[\delta_{Q}^{\epsilon}, \delta_{S}^{\eta}\right] \psi^{a} } & =\delta_{L}^{\omega} \psi^{a}+\delta_{D}^{\alpha} \psi^{a}+\delta_{R}^{m} \psi^{a}+\delta_{g}^{\lambda} \psi^{a},
\end{aligned}
$$

where

$$
\omega^{\mu \nu}=-\frac{i}{2} \bar{\epsilon} \Gamma^{\mu \nu} \eta, \quad \alpha=\frac{i}{2} \bar{\epsilon} \eta, \quad m^{i j}=\frac{i}{2} \bar{\epsilon} \Gamma^{i j} \eta, \quad \lambda=-\frac{i}{2} \bar{\epsilon}\left(\phi_{i}^{a} \Gamma^{i}+A_{\mu}^{a} \Gamma^{\mu}\right)(x \Gamma) \eta .
$$

In order to evaluate $\left[\delta_{S}^{\eta}, \delta_{S}^{\eta^{\prime}}\right] \psi$ in (59), we have used the equation of motion (37) and the Fierz identity (6). To show the equation (62), we have used the following Fierz identity

$$
-i \epsilon(\bar{\eta} \psi)-\frac{i}{2} \Gamma^{M} \eta\left(\bar{\psi} \Gamma_{M} \epsilon\right)=-\frac{i}{4} \psi(\bar{\epsilon} \eta)+\frac{i}{8} \Gamma^{M N} \psi\left(\bar{\epsilon} \Gamma_{M N} \eta\right)
$$

in addition to (37) and (6). This is proven in Appendix A.

\section{4. $\mathcal{N}=4$ superconformal algebra in $D=4$}

We may define generators $Q_{\alpha}, P_{\mu}, L_{\mu \nu}, R_{i j}, D, K_{\mu}$ and $S_{\alpha}$ by

$$
\begin{aligned}
& \delta_{Q}^{\epsilon} \Phi=-i\left[Q_{\alpha} \epsilon^{\alpha}, \Phi\right], \quad \delta_{P}^{a} \Phi=-i\left[a^{\mu} P_{\mu}, \Phi\right], \quad \delta_{L}^{\omega} \Phi=-i\left[\frac{1}{2} \omega^{\mu \nu} L_{\mu \nu}, \Phi\right], \\
& \delta_{R}^{m} \Phi=-i\left[\frac{1}{2} m^{i j} R_{i j}, \Phi\right], \quad \delta_{D}^{\alpha} \Phi=-i[\alpha D, \Phi], \quad \delta_{K}^{b} \Phi=-i\left[b^{\mu} K_{\mu}, \Phi\right], \\
& \delta_{S}^{\eta} \Phi=-i\left[S_{\alpha} \eta^{\alpha}, \Phi\right] .
\end{aligned}
$$

Following the method explained in section 2.1, we obtain the following commutation relations

$$
\begin{aligned}
{\left[L_{\mu \nu}, P_{\rho}\right] } & =i\left(\eta_{\nu \rho} P_{\mu}-\eta_{\mu \rho} P_{\nu}\right) \\
{\left[L_{\mu \nu}, L_{\rho \sigma}\right] } & =i\left(\eta_{\nu \rho} L_{\mu \sigma}-\eta_{\mu \rho} L_{\nu \sigma}-\eta_{\nu \sigma} L_{\mu \rho}+\eta_{\mu \sigma} L_{\nu \rho}\right) \\
{\left[R_{i j}, R_{k l}\right] } & =i\left(\delta_{j k} R_{i l}-\delta_{i k} R_{j l}-\delta_{j l} R_{i k}+\delta_{i l} R_{j k}\right) \\
{\left[D, P_{\mu}\right] } & =-i P_{\mu}, \quad\left[D, K_{\mu}\right]=+i K_{\mu}, \quad\left[L_{\mu \nu}, K_{\rho}\right]=i\left(\eta_{\nu \rho} K_{\mu}-\eta_{\mu \rho} K_{\nu}\right) \\
{\left[K_{\mu}, P_{\nu}\right] } & =2 i L_{\mu \nu}+2 i \eta_{\mu \nu} D
\end{aligned}
$$


and

$$
\begin{aligned}
{\left[Q_{\alpha}, L_{\mu \nu}\right] } & =-\frac{i}{2}\left(Q \Gamma_{\mu \nu}\right)_{\alpha}, \quad\left[Q_{\alpha}, R_{i j}\right]=-\frac{i}{2}\left(Q \Gamma_{i j}\right)_{\alpha}, \quad\left[D, Q_{\alpha}\right]=-\frac{i}{2} Q_{\alpha}, \\
{\left[S_{\alpha}, L_{\mu \nu}\right] } & =-\frac{i}{2}\left(S \Gamma_{\mu \nu}\right)_{\alpha}, \quad\left[S_{\alpha}, R_{i j}\right]=-\frac{i}{2}\left(S \Gamma_{i j}\right)_{\alpha}, \quad\left[D, S_{\alpha}\right]=+\frac{i}{2} S_{\alpha}, \\
{\left[K_{\mu}, Q_{\alpha}\right] } & =-i\left(S \Gamma_{\mu}\right)_{\alpha}, \quad\left[P_{\mu}, S_{\alpha}\right]=-i\left(Q \Gamma_{\mu}\right)_{\alpha}, \\
\left\{Q_{\alpha}, Q_{\beta}\right\} & =\frac{1}{2}\left(C \Gamma^{\mu} h_{+}\right)_{\alpha \beta} P_{\mu}, \quad\left\{S_{\alpha}, S_{\beta}\right\}=\frac{1}{2}\left(C \Gamma^{\mu} h_{-}\right)_{\alpha \beta} K_{\mu}, \\
\left\{Q_{\alpha}, S_{\beta}\right\} & =\frac{1}{4}\left(C \Gamma^{\mu \nu} h_{-}\right)_{\alpha \beta} L_{\mu \nu}-\frac{1}{2}\left(C h_{-}\right)_{\alpha \beta} D-\frac{1}{4}\left(C \Gamma^{i j} h_{-}\right)_{\alpha \beta} R_{i j} .
\end{aligned}
$$

It implies that the $\mathcal{N}=4 \mathrm{SYM}$ in $D=4$ is invariant under the $\mathcal{N}=4$ superconformal transformations in $D=4$, so that it's symmetry algebra is $\operatorname{psu}(2,2 \mid 4)$. The set of generators $\left\{P_{\mu}, L_{\mu \nu}, R_{i j}, Q_{\alpha}\right\}$ forms a subalgebra of $\operatorname{psu}(2,2 \mid 4)$, the $\mathcal{N}=4$ super-Poincaré algebra.

\section{Relation to supersymmetries in $\mathbf{A d S}_{5} \times \mathrm{S}^{5}$ background}

In this section, we shall rewrite the $\mathcal{N}=4$ superconformal algebra in $D=4$ given in (66) and (67) into the supersymmetry algebra of the $\mathrm{AdS}_{5} \times \mathrm{S}^{5}$ background. This gives a concrete map between generators, including fermionic ones.

First, we examine bosonic generators and show that they form so $(2,4) \times \operatorname{so}(6)$ of the isometry algebra of $\operatorname{AdS}_{5} \times S^{5}$. Let us define $J_{M N}(M, N=0,1,2,3,4, \sharp)$ by

$$
J_{\mu \nu}=L_{\mu \nu}, \quad J_{4 \sharp}=-D, \quad J_{\mu 4}=\frac{1}{2}\left(K_{\mu}-P_{\mu}\right), \quad J_{\mu \sharp}=\frac{1}{2}\left(K_{\mu}+P_{\mu}\right),
$$

and then $J_{M N}$ are shown to satisfy $\operatorname{so}(2,4)$

$$
\left[J_{M N}, J_{P Q}\right]=i\left(\eta_{N P} J_{M Q}-\eta_{M P} J_{N Q}-\eta_{N Q} J_{M P}+\eta_{M Q} J_{N P}\right)
$$

where $\eta_{M N}=\operatorname{diag}(-,+,+,+,+,-)$ with $\eta_{00}=\eta_{\sharp \sharp}=-1$. If we define $\hat{J}_{a b}$ and $\hat{P}_{a}$ $(a, b=0,1,3,4)$ by

$$
\hat{J}_{a b}=J_{a b}, \quad \hat{P}_{a}=J_{a \sharp},
$$

one finds

$$
\begin{aligned}
{\left[\hat{J}_{a b}, \hat{J}_{c d}\right] } & =i\left(\eta_{b c} \hat{J}_{a d}-\eta_{a c} \hat{J}_{b d}-\eta_{b d} \hat{J}_{a c}+\eta_{a d} \hat{J}_{b c}\right) \\
{\left[\hat{J}_{a b}, \hat{P}_{c}\right] } & =i\left(\eta_{b c} \hat{P}_{a}-\eta_{a c} \hat{P}_{b}\right), \quad\left[\hat{P}_{a}, \hat{P}_{b}\right]=i \hat{J}_{a b} .
\end{aligned}
$$

The $\hat{J}$ and $\hat{P}$ are generators of the Lorentz and translation symmetries in the $\mathrm{AdS}_{5}$ space.

The $R_{i j}(i, j=4,5,6,7,8,9)$ generates so(6). The $\hat{J}_{a^{\prime} b^{\prime}}$ and $\hat{P}_{a^{\prime}}\left(a^{\prime}, b^{\prime}=\right.$ $5,6,7,8,9)$ defined by

$$
\hat{J}_{a^{\prime} b^{\prime}}=R_{i j}, \quad \hat{P}_{a^{\prime}}=R_{4 a^{\prime}},
$$


are generators of the Lorentz and translation symmetries in $\mathrm{S}^{5}$

$$
\begin{aligned}
{\left[\hat{J}_{a^{\prime} b^{\prime}}, \hat{J}_{c^{\prime} d^{\prime}}\right] } & =i\left(\delta_{b^{\prime} c^{\prime}} \hat{J}_{a^{\prime} d^{\prime}}-\delta_{a^{\prime} c^{\prime}} \hat{J}_{b^{\prime} d^{\prime}}-\delta_{b^{\prime} d^{\prime}} \hat{J}_{a^{\prime} c^{\prime}}+\delta_{a^{\prime} d^{\prime}} \hat{J}_{b^{\prime} c^{\prime}}\right), \\
{\left[\hat{J}_{a^{\prime} b^{\prime}}, \hat{P}_{c^{\prime}}\right] } & =i\left(\eta_{b^{\prime} c^{\prime}} \hat{P}_{a^{\prime}}-\eta_{a^{\prime} c^{\prime}} \hat{P}_{b^{\prime}}\right), \quad\left[\hat{P}_{a^{\prime}}, \hat{P}_{b^{\prime}}\right]=-i \hat{J}_{a^{\prime} b^{\prime}} .
\end{aligned}
$$

Next we examine fermionic generators. Define $\mathcal{Q}_{I}(I=1,2)$ by

$$
\mathcal{Q}_{1} \equiv Q+S \Gamma_{4}, \quad \mathcal{Q}_{2} \equiv\left(Q-S \Gamma_{4}\right) \Gamma^{0123},
$$

and then one finds that (anti-)commutators including $Q$ or $S$ are rewritten as

$$
\begin{aligned}
{\left[\mathcal{Q}_{I}, \hat{J}_{a b}\right] } & =-\frac{i}{2} \mathcal{Q}_{I} \Gamma_{a b}, \quad\left[\mathcal{Q}_{I}, \hat{J}_{a^{\prime} b^{\prime}}\right]=-\frac{i}{2} \mathcal{Q}_{I} \Gamma_{a^{\prime} b^{\prime}} \\
{\left[\mathcal{Q}_{I}, \hat{P}_{a}\right] } & =\frac{i}{2} \epsilon_{I J} \mathcal{Q}_{J} \Gamma_{a} \mathcal{I}, \quad\left[\mathcal{Q}_{I}, \hat{P}_{a^{\prime}}\right]=-\frac{i}{2} \epsilon_{I J} \mathcal{Q}_{J} \Gamma_{a^{\prime}} \mathcal{I} \\
\left\{\mathcal{Q}_{I}, \mathcal{Q}_{J}\right\} & =\delta_{I J}\left(C \Gamma^{a} \hat{P}_{a}+C \Gamma^{a^{\prime}} \hat{P}_{a^{\prime}}\right) h_{+}-\frac{1}{2} \epsilon_{I J}\left(C \Gamma^{a b} \mathcal{I} \hat{J}_{a b}-C \Gamma^{a^{\prime} b^{\prime}} \mathcal{I} \hat{J}_{a^{\prime} b^{\prime}}\right) h_{+}
\end{aligned}
$$

where we have defined $\mathcal{I} \equiv \Gamma^{01234}$. The superalgebra (71), (73) and (75) is nothing but the supersymmetry algebra of the $\operatorname{AdS}_{5} \times \mathrm{S}^{5}$ background. In fact, after the replacement $J_{A B} \rightarrow i J_{A B}, P_{A} \rightarrow i P_{A}$ and $Q \rightarrow \frac{i}{\sqrt{2}} Q$, the superalgebra (71), (73) and (75) coincides with that in Ref. 4 which gives the $\mathrm{AdS}_{5} \times \mathrm{S}^{5}$ superalgebra ${ }^{5}$ in the ten-dimensional spinor notation.

Summarizing, we have shown that $\mathcal{N}=4$ superconformal algebra (66) and (67) is isomorphic to the superalgebra of $\mathrm{AdS}_{5} \times \mathrm{S}^{5}$, (71), (73) and (75).

Finally, we shall comment on the enhanced supersymmetries in the near-horizon limit of the D3-brane solution and the conformal supersymmetries of $\mathcal{N}=4 \mathrm{SYM}$. The $\mathrm{AdS}_{5} \times \mathrm{S}^{5}$ background is the near horizon limit of the D3-brane solution. The D3-brane solution preserves a half of 32 supersymmetries, while the $\mathrm{AdS}_{5} \times \mathrm{S}^{5}$ background does full 32 supersymmetries. In the near-horizon limit, 16 broken supersymmetries are restored so that the number of supersymmetries enhances to 32 .

For a D3-brane whose world-volume is extending along 0,1,2,3 directions, unbroken supersymmetry is specified by $\mathcal{Q}_{1}+\mathcal{Q}_{2} \Gamma^{0123}=0$, while broken supersymmetry by $\mathcal{Q}_{1}-\mathcal{Q}_{2} \Gamma^{0123}=0$. Now we know the concrete expression (74) which relates fermionic symmetries in the $\mathcal{N}=4 \mathrm{SYM}$ and those in the $\mathrm{AdS}_{5} \times \mathrm{S}^{5}$ background. We can see explicitly from (74) that

$$
\begin{aligned}
& \mathcal{Q}_{1}+\mathcal{Q}_{2} \Gamma^{0123}=Q+S \Gamma^{4}+\left(Q-S \Gamma^{4}\right)\left(\Gamma^{0123}\right)^{2}=2 S \Gamma^{4}, \\
& \mathcal{Q}_{1}-\mathcal{Q}_{2} \Gamma^{0123}=Q+S \Gamma^{4}-\left(Q-S \Gamma^{4}\right)\left(\Gamma^{0123}\right)^{2}=2 Q .
\end{aligned}
$$

This shows that the unbroken supersymmetry on D3-brane is related to the supersymmetry $Q$ of the $\mathcal{N}=4 \mathrm{SYM}$, while the enhanced supersymmetry in the near-horizon limit is noting but the conformal supersymmetry $S$ of the $\mathcal{N}=4$ SYM. 


\section{Summary and Discussion}

We have derived the $\mathcal{N}=4$ superconformal algebra from the symmetry transformations of fields in the $\mathcal{N}=4 \mathrm{SYM}$ in $D=4$. As the $\mathcal{N}=4 \mathrm{SYM}$ action in $D=4$ may be obtained from the $\mathcal{N}=(1,0)$ SYM action in $D=10$ via a dimensional reduction, the $\mathcal{N}=4$ supersymmetry in $D=4$ can be expressed by using a MW spinor in $D=10$. We found the map from generators of the $\mathcal{N}=4$ superconformal algebra to those of the super- $\mathrm{AdS}_{5} \times \mathrm{S}^{5}$ algebra. Especially we obtained the map (74) which relates fermionic generators: the supersymmetries $Q$ and the conformal supersymmetries $S$ in $\mathcal{N}=4 \mathrm{SYM}$ and the supersymmetries $\left(\mathcal{Q}_{1}, \mathcal{Q}_{2}\right)$ in the $\operatorname{AdS}_{5} \times \mathrm{S}^{5}$ background. It is obvious from this map that the conformal supersymmetries $S$ in the $\mathcal{N}=4 \mathrm{SYM}$ correspond to the supersymmetries which are broken by a D3-brane and are restored in the near horizon limit.

In this note, we have examined the classical symmetry of the $\mathcal{N}=4$ SYM. An important generalization is to discuss the quantum symmetry, such as the WardTakahashi identity. For the $\mathcal{N}=2 \mathrm{SYM}$, the anti-commutators of Noether supercharges are discussed in Ref. 6 and central charges are derived. Applying this method to the $\mathcal{N}=4 \mathrm{SYM}$, we find the following commutation relations

$$
\begin{aligned}
\left\{Q_{\alpha}, Q_{\beta}\right\} & =\frac{1}{2}\left(C \Gamma^{\mu} h_{+}\right)_{\alpha \beta} P_{\mu}+\frac{1}{2} Z_{\alpha \beta}, \\
\left\{S_{\alpha}, S_{\beta}\right\} & =\frac{1}{2}\left(C \Gamma^{\mu} h_{-}\right)_{\alpha \beta} K_{\mu}+\frac{1}{2} X_{\alpha \beta}, \\
\left\{Q_{\alpha}, S_{\beta}\right\} & =\frac{1}{4}\left(C \Gamma^{\mu \nu} h_{-}\right)_{\alpha \beta} L_{\mu \nu}-\frac{1}{2}\left(C h_{-}\right)_{\alpha \beta} D-\frac{1}{4}\left(C \Gamma^{i j} h_{-}\right)_{\alpha \beta} R_{i j}+\frac{1}{2} Y_{\alpha \beta},
\end{aligned}
$$

where $Z_{\alpha \beta}, X_{\alpha \beta}$ and $Y_{\alpha \beta}$ denote central charges of the form

$$
\begin{gathered}
Z_{\alpha \beta}=\int \mathrm{d}^{3} x\left[\left(C \Gamma^{\mu} h_{+}\right)_{\alpha \beta} A_{\mu}^{a} \Pi^{a 0}+\left(C \Gamma^{i} h_{+}\right)_{\alpha \beta} \phi_{i}^{a} \Pi^{a 0}+\cdots\right] \\
X_{\alpha \beta}=\int \mathrm{d}^{3} x\left[\left(C \Gamma^{\mu} h_{-}\right)_{\alpha \beta}\left(x^{2} \delta_{\mu}^{\nu}-2 x_{\mu} x^{\nu}\right) \Pi^{a 0} A_{\nu}^{a}+\left(C \Gamma^{i} h_{-}\right)_{\alpha \beta} x^{2} \Pi^{a 0} \phi_{i}^{a}+\cdots\right] \\
Y_{\alpha \beta}=\int \mathrm{d}^{3} x\left[-\left(C h_{-}\right)_{\alpha \beta} x^{\mu} A_{\mu}^{a} \Pi^{a 0}+\left(C \Gamma^{\mu \nu} h_{-}\right)_{\alpha \beta} x_{\mu} A_{\nu}^{a} \Pi^{a 0}\right. \\
\left.+\left(C \Gamma^{\rho i} h_{-}\right)_{\alpha \beta} x_{\rho} \phi_{i}^{a} \Pi^{a 0}+\cdots\right]
\end{gathered}
$$

The abbriviations represent terms including fermions. We have introduced $\Pi^{a \mu}$ defined by $\Pi^{a \mu} \equiv D_{\nu} F^{a \nu \mu}-g f^{a b c} \phi_{i}^{b} D^{\mu} \phi_{i}^{c}+\frac{i}{2} g f^{a b c} \bar{\psi}^{b} \Gamma^{\mu} \psi^{c}$. These central charges vanish if the equation of motion $\Pi^{a \mu}=0$ (and the fermionic counterpart) is used. It is interesting to examine a realization of these central charges in the AdS side by using the map derived in this note. We hope to report the complete form of these central charges and discuss this point in the next work. ${ }^{7}$

Another important generalization is to examine supersymmetries in $\mathrm{AdS}_{4} / \mathrm{CFT}_{3}$. It is known that both the $\mathcal{N}=6$ superconformal algebra of the $\mathcal{N}=6$ Chern-Simons-matter (CSM) theory (the ABJM model ${ }^{8}$ ) and the super-isometry algebra of the $\mathrm{AdS}_{4} \times \mathbb{C P}^{3}$ background are osp(6|4). As in the $\mathrm{AdS}_{5} / \mathrm{CFT}_{4}$ case, we 
can show that the conformal supersymmetries in the $\mathcal{N}=6$ CSM are supersymmetries which are broken by an M2-brane and are restored in the near horizon limit. We will report this subject in near future.

\section{Appendix A. Fierz identities in $D=10$}

The spin-dimension in $D=10$ is 32 . The following matrices composed of Gammamatrices $\Gamma^{M}$ form a complete set of $32 \times 32$ matrices

$$
\begin{aligned}
\lambda^{A}=\{ & 1, \Gamma^{M}, \frac{i}{\sqrt{2 !}} \Gamma^{M N}, \frac{i}{\sqrt{3 !}} \Gamma^{M_{1} \cdots M_{3}}, \frac{1}{\sqrt{4 !}} \Gamma^{M_{1} \cdots M_{4}}, \frac{1}{\sqrt{5 !}} \Gamma^{M_{1} \cdots M_{5}}, \\
& \left.\frac{i}{\sqrt{6 !}} \Gamma^{M_{1} \cdots M_{6}}, \frac{i}{\sqrt{7 !}} \Gamma^{M_{1} \cdots M_{7}}, \frac{1}{\sqrt{8 !}} \Gamma^{M_{1} \cdots M_{8}}, \frac{1}{\sqrt{9 !}} \Gamma^{M_{1} \cdots M_{9}}, \frac{i}{\sqrt{10 !}} \Gamma^{M_{1} \cdots M_{10}}\right\} .
\end{aligned}
$$

The matrices $\lambda_{A}$ are defined by $\operatorname{tr} \lambda^{A} \lambda_{B}=32 \delta_{B}^{A}$.

Let $\phi_{i}(i=1,2,3,4)$ be Grassmann-even spinors in $D=10$, and let $\Lambda_{1}$ and $\Lambda_{2}$ be $32 \times 32$ matrices, and then we have the identity ${ }^{\mathrm{b}}$

$$
\left(\bar{\phi}_{1} \Lambda_{1} \phi_{2}\right)\left(\bar{\phi}_{3} \Lambda_{2} \phi_{4}\right)=\frac{1}{32} \sum_{A}\left(\bar{\phi}_{1} \lambda^{A} \phi_{4}\right)\left(\bar{\phi}_{3} \Lambda_{2} \lambda_{A} \Lambda_{1} \phi_{2}\right) .
$$

When $\phi_{2}=\phi_{3}=\phi_{4} \equiv \phi$ and $\phi_{i}$ have the same chirality $h_{+} \phi_{i}=\phi_{i}$, we have

$$
\left(\bar{\phi}_{1} \Gamma^{M} \phi\right)\left(\bar{\phi} \Gamma_{M} \phi\right)=0 \text {. }
$$

This is nothing but the Fierz identity (6).

Finally we derive the Fierz identity (64). Let $\epsilon, \psi$ and $\eta$ be Grassmann-odd spinors of $h_{+} \epsilon=\epsilon, h_{+} \psi=\psi$ and $h_{-} \eta=\eta$. For our purpose, we derive two Fierz identities,

$$
\epsilon \bar{\eta} \psi=-\frac{1}{16}\left[\psi \bar{\eta} \epsilon-\frac{1}{2 !} \Gamma^{M N} \psi \bar{\eta} \Gamma_{M N} \epsilon+\frac{1}{4 !} \Gamma^{M_{1} \cdots M_{4}} \psi \bar{\eta} \Gamma_{M_{1} \cdots M_{4}} \epsilon\right],
$$

and

$$
\begin{aligned}
\Gamma^{M} \eta \bar{\epsilon} \Gamma_{M} \psi=-\frac{1}{16}[ & D \psi \bar{\epsilon} \eta-\frac{1}{2 !}(D-4) \Gamma^{M N} \psi \bar{\epsilon} \Gamma_{M N} \eta \\
& \left.+\frac{1}{4 !}(D-8) \Gamma^{M_{1} \cdots M_{4}} \psi \bar{\epsilon} \Gamma_{M_{1} \cdots M_{4}} \eta\right]
\end{aligned}
$$

where $D=10$. The Fierz identity (64) can be obtained as $-i(\mathrm{~A} .4)+\frac{i}{2}(\mathrm{~A} .5)$.

\section{Acknowledgments}

The authors would like to thank Takanori Fujiwara, Yoshifumi Hyakutake, Hyeonjoon Shin, Haruya Suzuki and Kentaroh Yoshida for useful comments.

\footnotetext{
${ }^{\mathrm{b}}$ The right hand side acquires an extra minus sign for Grassmann-odd spinors.
} 


\section{References}

1. G. 't Hooft, Nucl. Phys. B 72 (1974) 461.

2. J. M. Maldacena, Int. J. Theor. Phys. 38 (1999) 1113 [Adv. Theor. Math. Phys. 2 (1998) 231] [hep-th/9711200].

3. S. S. Gubser, I. R. Klebanov and A. M. Polyakov, Phys. Lett. B 428 (1998) 105 [hepth/9802109].

E. Witten, Adv. Theor. Math. Phys. 2 (1998) 253 [hep-th/9802150].

4. M. Hatsuda, K. Kamimura and M. Sakaguchi, Nucl. Phys. B 632 (2002) 114 [hepth/0202190].

5. R. R. Metsaev and A. A. Tseytlin, Nucl. Phys. B 533 (1998) 109 [hep-th/9805028].

6. K. Fujikawa and K. Okuyama, Nucl. Phys. B 521 (1998) 401 [hep-th/9708007].

7. M. Sakaguchi, F. Takeuchi and K. Yoshida, work in progress.

8. O. Aharony, O. Bergman, D. L. Jafferis and J. Maldacena, JHEP 0810 (2008) 091 [arXiv:0806.1218 [hep-th]]. 